\title{
Visualization of 3-Dimensional Vectors in a Dynamic Embryonic System-WormGUIDES
}

\author{
Eric Wang1,2, Anthony Santella3 ${ }^{3}$ Zi Wang1, Dali Wang1,4, Zhirong Bao ${ }^{3}$ \\ ${ }^{1}$ Department of Electric Engineering and Computer Science, University of Tennessee, Knoxville, TN, USA \\ ${ }^{2}$ Coding Club, Farragut High School, Knoxville, TN, USA \\ ${ }^{3}$ Developmental Biology Program, Memorial Sloan Kettering Cancer Center, New York, NY, USA \\ ${ }^{4}$ Environmental Science Division, Oak Ridge National Laboratory, Oak Ridge, TN, USA \\ Email: ericwang2000@outlook.com, santella@mskcc.org, zwang84@vols.utk.edu,dwang7@utk.edu, baoz@mskcc.org
}

How to cite this paper: Wang, E., Santella, A., Wang, Z., Wang, D.L. and Bao, Z.R. (2017) Visualization of 3-Dimensional Vectors in a Dynamic Embryonic System-WormGUIDES. Journal of Computer and Communications, 5, 70-79.

https://doi.org/10.4236/jcc.2017.512008

Received: September 19, 2017

Accepted: October 27, 2017

Published: October 30, 2017

Copyright $\odot 2017$ by authors and Scientific Research Publishing Inc. This work is licensed under the Creative Commons Attribution International License (CC BY 4.0).

http://creativecommons.org/licenses/by/4.0/ Open Access

\begin{abstract}
WormGUIDES is an open-source dynamic embryonic system designed to facilitate global understanding of cellular decisions in the developing nervous system of the nematode $C$. elegans. WormGUIDES was designed to allow investigation and exploration of the observational results of the C. elegans life cycle from laboratory experiments. In the process of a mechanistic C. elegans model development, some functionalities of WormGUIDES needed to be enhanced to support model validation and verification. In this study, a new way to visualize 3-dimentional vectors within WormGUIDES was investigated and presented. Then, the practical values of this method were demonstrated by visualizing two biologically significant directions (i.e., division orientation and cell polarity) of individual embryonic cells in C. elegans. Lastly, a mathematic approach was designed to illustrate the differences between these two sets of vectors and provide easy indications of the location of these individual cells that have large data discrepancies within the $C$. elegans embryonic system.
\end{abstract}

\section{Keywords}

Embryonic Data Visualization, WormGUIDES, Workflow, Software Architecture, Division Orientation, Cell Polarity, C. elegans

\section{Introduction}

In the past several years, a consortium of biologists, computer scientists, and microscopists from the Memorial Sloan Kettering Cancer Center, Yale University, the University of Connecticut, and the National Institute of Health have worked together to create a novel systems-level resource that will facilitate examination of cellular decisions in the developing nervous system of the nema- 
tode $C$. elegans. This resource, used for global understanding in dynamic embryonic systems, is WormGUIDES [1]. WormGUIDES was solely a mobile app until 2016, when a desktop version of WormGUIDES was released with detailed information on cell shapes, note-taking, and sharing functionality, as well as single-cell information summaries [2]. Along with current development of agent-based embryogenesis modeling [3] [4], we would like to extend the WormGUIDES functionality for model verification and validation. This process is necessary in order to achieve an efficient way of comparing any $3 \mathrm{D}$ vectors through visualization. In this paper, we first summarize the software architecture of WormGUIDES, and then present a method to visualize the 3-dimentional vectors with RGB values. Finally, we demonstrate the practical use of this method to visualize two sets of 3-dimentional vectors with significant biological meanings: division orientation and cell polarity. The experiment can be extended to other vectors to facilitate further model calibration and verification.

\section{Software Architecture of WormGUIDES}

\subsection{Computational Platform and Software Architecture}

The source code for the desktop version of WormGUIDES is located on GitHub for free download [5]. The computational platforms used in the study are a MacBook Air and a workstation at MSKCC. The detailed WormGUIDES installation instructions are available online [6].

Currently, there are two important functions embedded within WormGUIDES. One of these functions is designed to contain and display the C. elegans lineage tree information from previous experiments [7] and after gene mutation \& manipulation [8]. The other important function is to allow access to and visualize the connectome, the complete neural connectivity record which is uniquely available for C. elegans [9].

Additionally, WormGUIDES contains several utilities to support user search and query. The WormGUIDES interface is illustrated in Figure 1.

As shown in Figure 1, the WormGUIDES software is designed to provide intuitive and elegant representations of $C$. elegans's information. WormGUIDES's main interface contains many functions to improve the accessibility of underlying data. Beginning with the first tab on the left side of WormGUIDES, Stories are user created shareable annotations, meaning that they are text that offers more specific insight into embryonic events. They appear on the active screen on the right when selected by the user. Next to Stories, the Coloring and Display tab can be used to find cells or multicellular structures, and defines many different ways of selecting and coloring cells based on different attributes. Under the main $3 \mathrm{D}$ window a scroll bar allows the user to navigate any part of the embryo at any point of its life from 20 mins after the first cell cleavage to $~ 379$ mins later when spontaneous muscle movements begin. Under the scroll bar, when a cell is clicked on the active screen, the Information Box provides more detailed information on the functionality of the cell and the cell's descendants. Additionally, it provides more information on the current active story. 


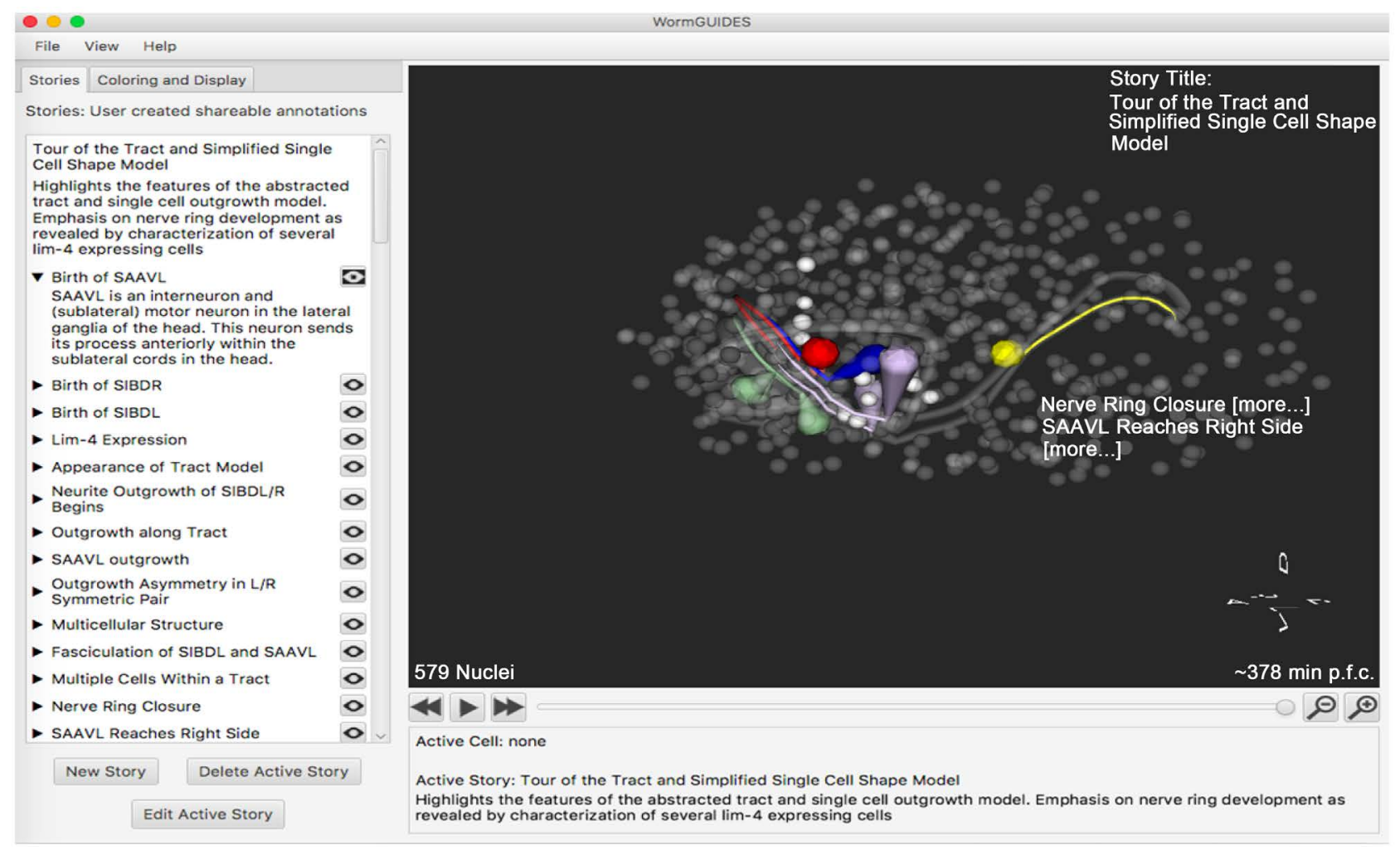

Figure 1. The main interface of WormGUIDES.

\subsection{Data Visualization Procedure within WormGUIDES System}

WormGUIDES provides the intuitive creation and sharing of interactive visualizations. Users can create custom color schemes to highlight features of interest. Multiple color layers can be combined to create an interactive 4D illustration of key events or features. This view of the embryo can then be shared with others encoded in a URL text string. This functionality can be used to visualize arbitrary single cell data superimposed on the model by computing a mapping from data into RGB. A color space and outputting a URL which assigns each cell in the embryo a unique data-driven color.

\subsection{The Visualization of 3D Vectors}

In the agent-based modeling for C. elegans embryogenesis, several important biological concepts (such as the previously mentioned examples of division orientation and cell polarity) are represented in the format of vectors. Therefore, a good visualization of $3 \mathrm{D}$ vectors with RGB values is necessary. To convert the vector directions into RGB values, we must first setup a defined range for each vector to map onto the RGB value. Since the magnitude of the vector is not necessary to find the division orientation, we can normalize the XYZ components of vectors. By normalizing the vectors, we set the max value for the XYZ vectors at 1 and the min at -1 , therefore allowing the RGB values, from 0 to 255 , to be easily mapped onto the vector values. We visualize these $3 \mathrm{D}$ vectors in WormGUIDES using a simple algorithm. By associating the $\mathrm{X}, \mathrm{Y}$, and $\mathrm{Z}$ directions with 
R, G, and B values, respectively, we can create a colored system of cells in WormGUIDES that defines in which direction the mother cell will split into its daughters.

\section{Practical Applications of Data Visualization}

An important example of a vector to visualize is cell division orientation, defined as the direction in which a parent cell splits into two daughter cells. Cell division orientation is important for morphogenesis, cell fate, and tissue homeostasis. In this section, we use our color schemes to visualize the division of orientation, measured from tracked cell positions. In $C$. elegans embryogenesis, the division orientation is closely related to another concept, cell polarity, which is defined as "the asymmetric organization of several cellular components, including its plasma membrane, cytoskeleton or organelles" [10]. Cell polarity causes asymmetric organization within a cell, such as localization of molecules. Cell polarity impacts the division orientation [11] since the mitotic spindle can be oriented based on the asymmetric localization of regulators, such as PAR proteins, or the Wnt signaling pathway. However, unlike division orientation, cell polarity caused by many factors and cannot be easily modeled. As a result, we first make several assumptions to derive a cell polarity from a division orientation. Then we can visualize the cell polarity and quantify the differences between division orientation and cell polarity to identify the sources of these differences.

\subsection{Calculation and Visualization of Division Orientation from Observation}

A MatLab program is created to calculate the division orientations from observational datasets, derived directly from the microscopic images from Dr. Bao's lab. Each dataset contains the cell list at a specific timestamp during the observation. The interval of observation is around 60 seconds. The pseudo code of division orientation calculation is illustrated in Figure 2. First, we load the tracked

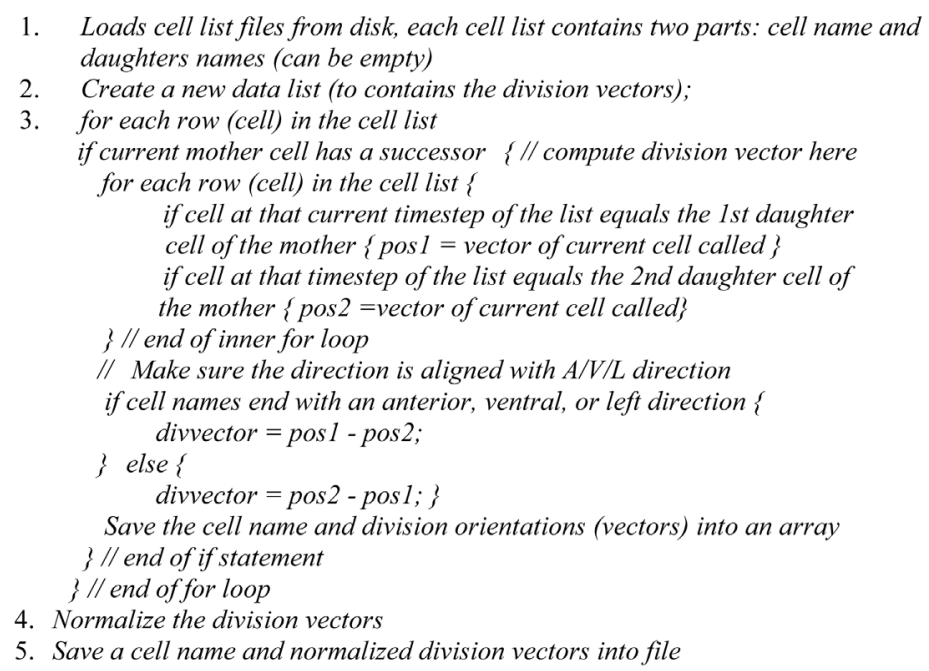

Figure 2. Pseudo code for division orientation calculation. 
cell locations. Then, we align the XYZ axes with the individual body axis of cell separately: $\mathrm{X}$ axis for the Anterior-posterior (AP) direction, the $\mathrm{Y}$ axis for the Ventral-dorsal (DV) direction, and the $\mathrm{Z}$ axis for the Left-right (LR) direction. The pseudo code of division orientation calculation is illustrated in Figure 2.

Following the procedure described in Section 2.4, the collection of visualization results is shown in Figure 3. As illustrated, each dot represents one nuclei in the embryo. The colors of the dots in the images above each represent the division orientation of one of the cells, which is derived from the observational dataset. Each image is representative of a different point of time in the embryo's development. From these images, we observe that the division orientation of $\mathrm{AB}$ related cells at each generation have similar colors, especially at the early development phase (before the 220 minutes after the beginning of embryo growth). This observation leads us to an assumption that the dominant driving force in each generation of $\mathrm{AB}$-related cell development may be the same. It can also be used to estimate the cell polarity in the cell development.

\subsection{Calculation and Visualization of Cell Polarity of Cells within AB Sub-Lineage}

In our study, we define cell polarity as the main factor for determining division orientation. As we previously mentioned in Section 3, it is difficult to model the cell polarity directly from observation. However, in Section 3.1 we observed that the division orientation of $\mathrm{AB}$ related cells at each generation have similar directions at the early development phase. As a result, we may assume cell polarity for each cell in the $\mathrm{AB}$ sub-lineage tree in a generation is the same. The cell polarity of all the cells of same generation within $\mathrm{AB}$ sub-lineage tree is thereby calculated by averaging the division orientations for the generation of cells. Then, adding the $\mathrm{X}, \mathrm{Y}$, and $\mathrm{Z}$ components of the vectors to get the main vector, normalizing this main vector, and splitting it back into components should allow the cell polarity to be easily mapped onto its respective RGB values.

As illustrated in Figure 4, the cell polarity within $A B$ lineage changes from generation to generation. The color of cell polarity in the first image is light green, which means the direction more aligns with $\mathrm{Z}$ axis, while the color of cell polarity in the second image is orange, which means the direction more aligns with X-Y plane. It is a very rapid change. From the third image, the colors of cell polarity vary within the range of orange and pink/red, which shows the direction is still aligned with the $\mathrm{X}-\mathrm{Y}$ plane. The comparison between Figure 3 and Figure 4 shows differences of these two sets of vectors, so that we may need to reevaluate of our methods and assumptions of cell polarity calculations. In the next section, we would like to quantify these differences to identify the sources for the major data discrepancy.

\subsection{Quantification of Differences between Observed Division Orientation and Calculated Cell Polarity}

To find the differences between the observed division orientation and calculated 

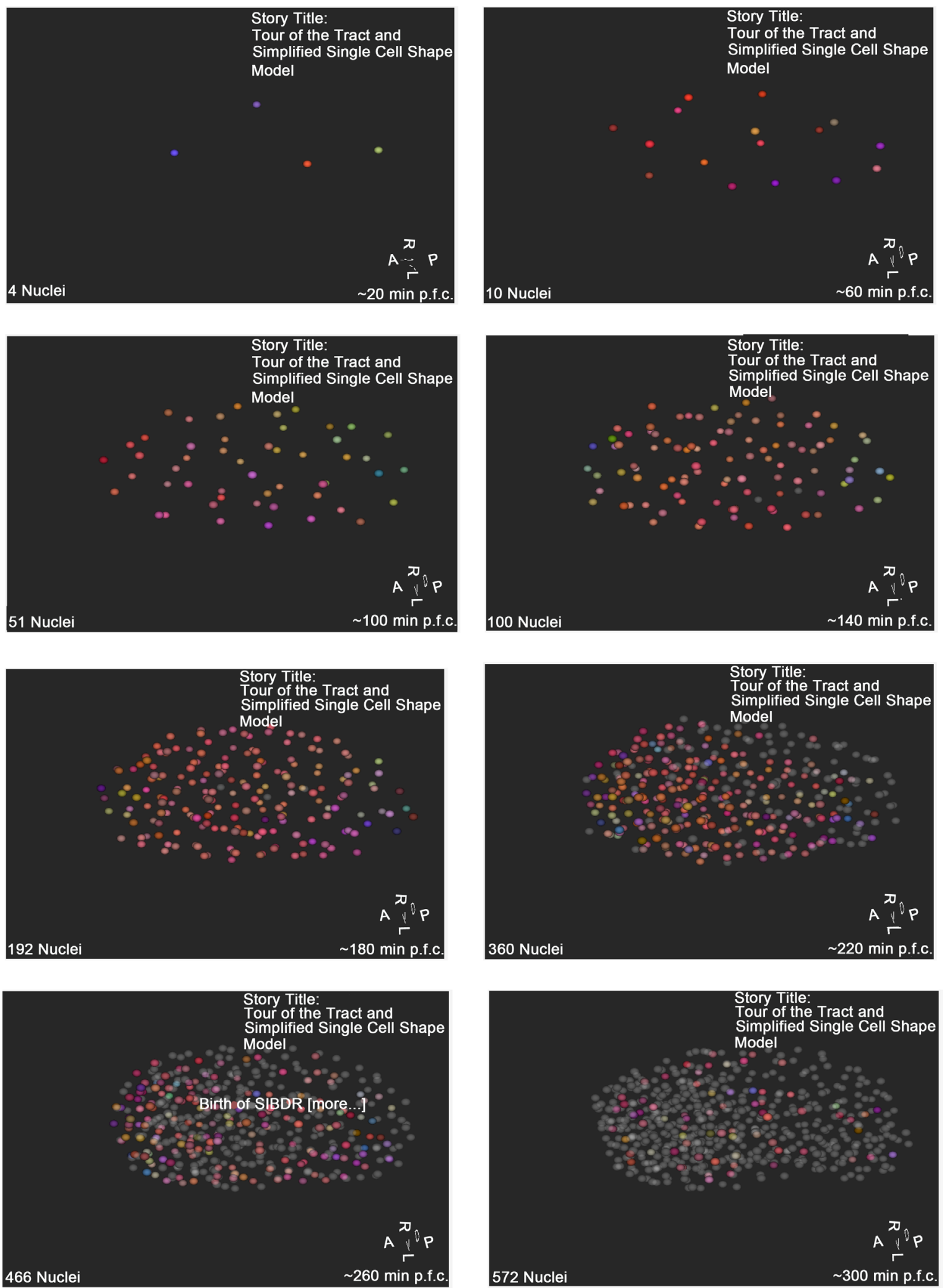

Figure 3. The division orientations of the cells at different times of the embryo's development. The red and orange color majority reveals that the majority of the cell divisions are in the Anterior/Posterior direction and less so in the other Right/Left (Yellow) and Ventral/Dorsal (Blue) directions. The first image, top left, is at 20 mins after embryo development, followed by increments of 40 mins, up to 300 mins on the bottom right image. The gray cells represent fully mature cells that do not further divide. 

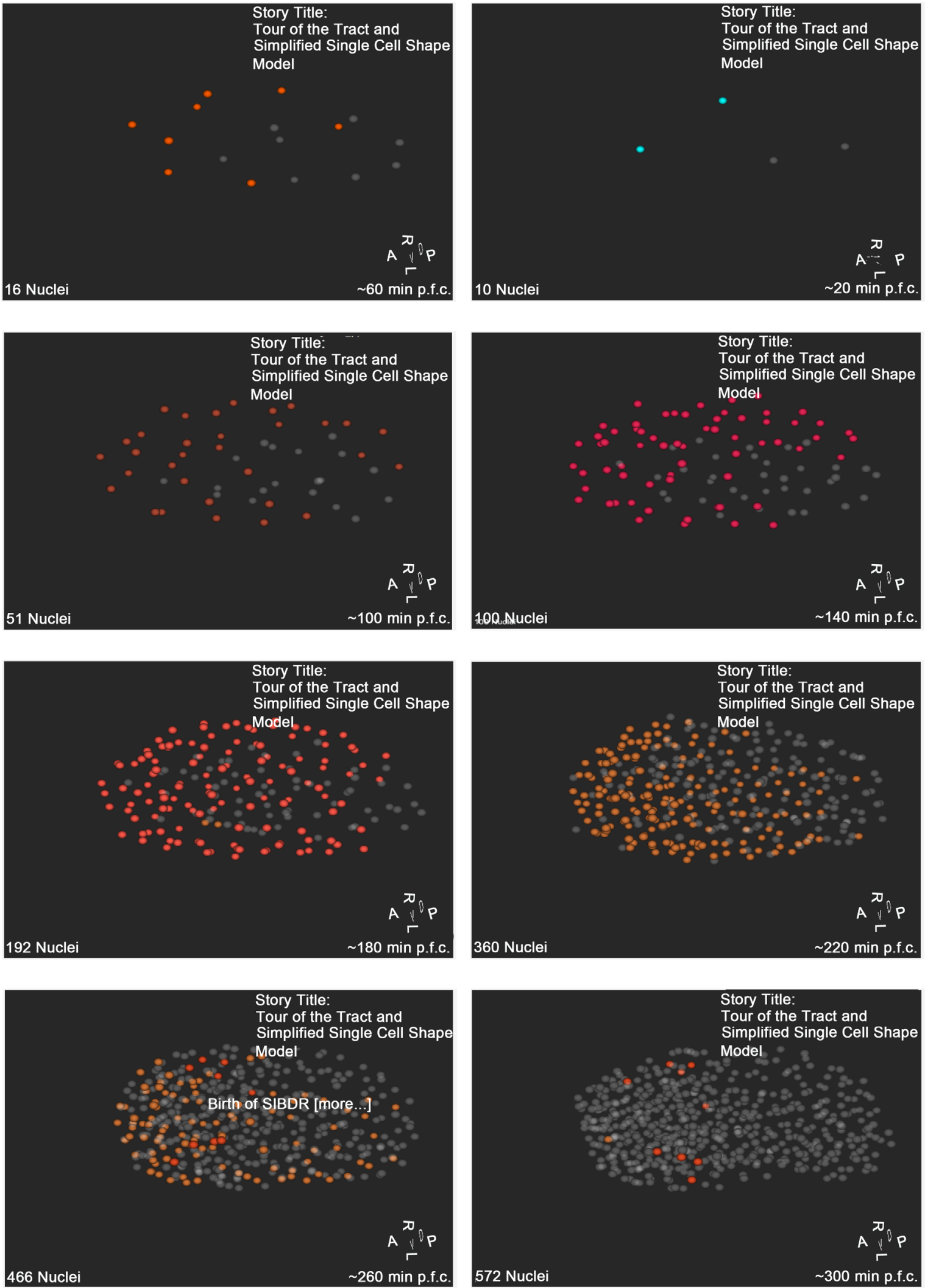

Figure 4. The visualization of cell polarities of cells within the AB sub-lineage. The rest cells are not relevant and are colored in gray. The first image, top left, is at 20 minutes after embryo development, followed by increments of 40 minutes (left and then down) up to 300 minutes on the bottom right image. 
Cell Polarity, a DotProduct function was developed in MatLab to quantify the discrepancy of these two set of vectors. Each vector is associated with individual cell that can be used to link the vectors between these two datasets. Since only the direction, not the quantity, of the vector is of interest, all the vectors are normalized first before the dot production operation. The results are easy to understand: the closer the value of dot product is to 1 , the closer the two individual vectors match. For the demonstration purpose, the top 10 cells with the large differences between these two vectors are listed in Table 1. The complete comparison results are available in Dropbox [12].

As shown in Table 1, the two vectors associated with cell $A B$ alpppaap have the biggest errors, the negative value (close to -1 ) meaning that these two vectors have almost completely opposite directions. It is also noticeable the large differences occurred at a later stage of development. The result also reiterates that at the early development phase the division orientations of $\mathrm{AB}$ related cells have similar division orientation.

\section{Conclusions and Discussions}

WormGUIDES is an open-source dynamic embryonic system developed by collaborations between Memorial Sloan Kettering Cancer Center, Yale, University of Connecticut Medical Center and the National Institute of Health. WormGUIDES can support the examination of cellular divisions in the developing nervous system of the nematode $C$. elegans. To facilitate mechanistic embryonic system model development, we need a visualization tool to identify the locations $\&$ ranges of $3 \mathrm{D}$ vector data and the discrepancy between $3 \mathrm{D}$ vectors datasets. In this paper, we have presented a new way to visualize $3 \mathrm{D}$ vectors within WormGUIDES. We have laid out the implementation details and demonstrated the functionality by visualizing both the division orientation and the calculated cell polarity of individual embryonic cells in $\mathrm{AB}$ sub-lineage of $C$. elegans. Different

Table 1. The differences (Dot Product) between the Division Orientation and Cell Polarity of Cells within the AB Sub-lineage.

\begin{tabular}{cccc}
\hline Cell Name & Division Orientation & Cell Polarity & Dot Product \\
\hline “ABalpppaap" & {$[-0.724,0.634,0.272]$} & {$[0.945,-0.281,-0.168]$} & -0.91 \\
“ABalaaappr" & {$[-0.862,-0.503,-0.072]$} & {$[0.945,-0.281,-0.168]$} & -0.66 \\
“ABalpppapa" & {$[-0.212,0.954,0.212]$} & {$[0.945,-0.281,-0.168]$} & -0.50 \\
“ABalppppaa" & {$[-0.456,-0.836,-0.304]$} & {$[0.945,-0.281,-0.168]$} & -0.14 \\
“ABalaapppa" & {$[-0.323,-0.889,0.323]$} & {$[0.945,-0.281,-0.168]$} & -0.11 \\
“ABalaaaarr" & {$[-0.351,-0.935,-0.058]$} & {$[0.945,-0.281,-0.168]$} & -0.06 \\
“ABalaaapa" & {$[0.316,0.949,0.000]$} & {$[0.928,-0.370,-0.032]$} & -0.06 \\
“ABalpaaa" & {$[-0.580,-0.811,0.077]$} & {$[0.833,-0.551,0.050]$} & -0.03 \\
“ABarapaapa" & {$[-0.236,-0.943,0.236]$} & {$[0.945,-0.281,-0.168]$} & 0.00 \\
“ABprppp" & {$[-0.508,-0.860,-0.039]$} & {$[0.847,-0.498,-0.187]$} & 0.01 \\
\hline
\end{tabular}


hypotheses will be proposed in the C. elegan's embryogenesis model development, especially for the cells that divide in the directory of D/V or LR, instead of AP. Therefore, this visualization and discrepancy quantification will provide information to facilitate the identification of major factors for regulating cell division orientation. In addition, this tool can be used to provide intuitive access to information on the location properties of these individual cells with large data discrepancies.

\section{Acknowledgements}

This study is supported by an NIH research project grants (R01GM097576 and 2R01GM097576). Research in the Bao lab is also supported by an NIH center grant to MSKCC (P30CA008748).

\section{References}

[1] Santella, A., Catena, R., Kovacevic, I., Shah, P., Yu, Z.D., Marquina-Solis, J., Kumar, A., et al. (2015) WormGUIDES: An Interactive Single Cell Developmental Atlas and Tool for Collaborative Multidimensional Data Exploration. BMC Bioinformatics, 16, 189. https://doi.org/10.1186/s12859-015-0627-8

[2] C. elegans Tropic Meeting: Neuronal Development, Synaptic Function and Behavior, CeNeuro 2016, and Nagoya BNC Symposium.

http://www.bio.nagoya-u.ac.jp/ ceneuro2016/

[3] Wang, Z., Ramsey, B.J., Wang, D.L., Wong, K., Li, H.S., Wang, E. and Bao, Z.R. (2016) An Observation-Driven Agent-Based Modeling and Analysis Framework for C. elegans Embryogenesis. PLoS ONE, 11, e0166551. https://doi.org/10.1371/journal.pone.0166551

[4] Wang, Z., Wang, D.L., Li, H.S. and Bao, Z.R. (2017) Cell Neighbor Determination in the Metazoan Embryo System. Proceedings of the 8th ACM International Conference on Bioinformatics, Computational Biology, and Health Informatics, Boston, MA, August 2017, 305-312.

[5] WormGUIDES Source Code in GitHUB. https://github.com/tangydoris/WormGUIDES

[6] Wang, E. WormGUIDES Installation Guide with Eclipse. Available in Dropbox at https://www.dropbox.com/s/v9uv0m109vsrhb4/WormGUIDES_installation_revise d_v1.0.docx?dl=0

[7] Bao, Z., Murray, J.I., Boyle, T., Ooi, S.L., Sandel, M.J. and Waterston, R.H. (2006) Automated Cell Lineage Tracing in Caenorhabditis elegans. Proceedings of the National Academy of Sciences of the United States of America, 103, 2707-2712. https://doi.org/10.1073/pnas.0511111103

[8] Du, Z., Santella, A., He, F., Shah, P.K., Kamikawa, Y. and Bao, Z.R. (2015) The Regulatory Landscape of Lineage Differentiation in a Metazoan Embryo. Developmental Cell, 34, 592-607. https://doi.org/10.1016/j.devcel.2015.07.014 http://www.sciencedirect.com/science/article/pii/S1534580715004876

[9] White, J.G., Southgate, E., Thomson, J.N. and Brenner, S. (1986) The Structure of the Nervous System of the Nematode Caenorhabditis elegans. The Mind of a Worm. Philosophical Transactions of the Royal Society London, 314, 1-340. https://doi.org/10.1098/rstb.1986.0056 
[10] The Definition of Cell Polarity at Nature.com. https://www.nature.com/subjects/cell-polarity

[11] Cowan, C.R. and Hyman, A.A. (2004) Asymmetric Cell Division in C. elegans: Cortical Polarity and Spindle Positioning. Annual Review of Cell and Developmental Biology, 20, 427-453.

https://doi.org/10.1146/annurev.cellbio.19.111301.113823

[12] Comparison of Division Orientation and Cell Polarity of Individual Cell within AB-Lineage. Available in Dropbox at

https://www.dropbox.com/s/324p2apyml16dnk/DPErrorData.xlsx?dl=0 